05,11

\title{
Динамические и статические свойства негейзенберговского магнетика со сложной межионной анизотропией
}

\author{
(ㄱ Г.А. Гореликов, О.А. Космачев, А.В. Кривцова, Ю.А. Фридман \\ Крымский федеральный университет им. В.И. Вернадского, \\ Симферополь, Россия \\ ๑ E-mail: yuriifridman@gmail.com \\ (Поступила в Редакцию 11 июля 2016 г.)
}

Исследовано влияние анизотропного обменного взаимодействия на фазовые состояния негейзенберговского ферромагнетика со спином магнитного иона $S=1$. Показано, что в зависимости от соотношений параметров анизотропного обменного взаимодействия, а также их знака в системе реализуется либо двухосный негейзенберговский магнетик, либо изинговский. Изучены динамические свойства системы в окрестностях ориентационных фазовых переходов, а также фазовых переходов по модулю магнитного момента. Построены фазовые диаграммы системы при различных соотношениях материальных параметров.

Работа выполнена при финансовой поддержке РФФИ (проекты № 16-02-00069а и № 16-32-00098 мол_а).

DOI: 10.21883/FTT.2017.03.44159.289

\section{1. Введение}

Интерес к изучению новых фазовых состояний спиновых систем магнетиков растет в течение последних двух десятков лет [1-8]. Для изотропных магнетиков при наличии высших по спинам обменных слагаемых типа $\left(\mathbf{S}_{1} \mathbf{S}_{2}\right)^{n}, n>1$ возможна реализация не только фаз с магнитным порядком, то есть с отличным от нуля средним значением спина (дипольного параметра порядка $\langle\mathbf{S}\rangle)$, но также и фаз с $\langle\mathbf{S}\rangle=0$, в которых спонтанное нарушение вращательной симметрии обусловлено средними значениями спиновых мультиполей, например, квадрупольными средними $S^{i j}\left\langle S^{i} S^{j}+S^{j} S^{i}\right\rangle / 2$. Вначале такое состояние, названное спиновым нематиком (SN), было найдено для магнетиков со спином $S=1$. Для этой системы найдена также ортогональная нематическая (ON); для которой ориентации главной оси квадрупольного эллипсоида перпендикулярны для разных подрешеток $[1,2,5]$. Геометрическим образом SN-фазы является квадрупольный эллипсоид, представляющий симметричный тензор второго ранга $S^{i j}$ (средние с более высокими степенями спинов тривиальны), в основном состоянии этот эллипсоид является эллипсоидом вращения и симметрия состояния на узле есть $C_{\infty}$. Подобные состояния для высших спинов $S>1$ изучены слабее, но для них обнаружены качественно новые эффекты, отсутствующие для случая $S=1$. Для спина $S=3 / 2$ найдены фазы с $\langle\mathbf{S}\rangle=0$, для которых симметрия относительно отражения времени нарушена за счет нетривиальных свойств трехспиновых средних [9], из которых можно составить планарный вектор псевдоспина $\boldsymbol{\sigma}[10]$. В силу этого для спина $S=3 / 2$ возможно существование антинематической (AN) фазы, в которой направления $\boldsymbol{\sigma}$ в двух подрешетках антипараллельны [10]. Для чисто изотропной модели магнетиков со спинами $S=1$ и $S=3 / 2$ в приближении среднего поля с взаимодействием ближайших соседей на решетке, допускающей разбиение на две подрешетки, построена полная фазовая диаграмма (далее мы проводим анализ только с учетом тех же приближений).

Наряду с исследованием как статических, так и динамических свойств изотропных спиновых систем, представляет интерес исследование обменно-анизотропных моделей. Одна из самых простых моделей, содержащая в гамильтониане тензорные взаимодействия - это обобщение изинговской модели с учетом биквадратичного взаимодействия - т.н. модель Блюма-ЭмериГриффитца [11]. Эта модель была предложена для описания термодинамических свойств смеси $\mathrm{He}(\mathrm{III})$ и $\mathrm{He}(\mathrm{IV})$ в окрестности критической точки. В работах $[12,13]$ обобщена модель Блюма-Эмери-Грифитса на негейзенберговские магнетики с $S=1$. Было показано, что учет анизотропного обменного взаимодействия (типа модели Изинга или $X Y$-модели) оказывает существенное влияние обменной анизотропии на динамику системы, а также формирование специфических фазовых состояний. В работах $[14,15]$ исследовалось влияние как межионной, так и одноионной анизотропий на свойства негейзенберговских магнетиков. Однако указанные выше исследования проводились для наиболее простых моделей межионной анизотропии.

Не вдаваясь в природу возникновения межионной анизотропии магнитных взаимодействий, целью данной работы является изучение влияния анизотропного межионного взаимодействия, более сложного, чем в [12-15], на фазовые состояния негейзенберговского ферромагнетика со спином магнитного иона $S=1$. Вполне ожидаемо, что исследование более сложных систем будут приводить к выявлению новых необычных свойств магнетиков. Рассмотрение дополнительных возможностей симметрии билинейного и биквадратичного обменного взаимодействий, по всей видимости, приводит к более разнообразной структуре фазовых состояний, а также динамических свойств системы. 


\section{2. Модель}

Рассмотрим трехмерный ферромагнетик со спином магнитного иона единица. Такой выбор величины спина позволяет учесть, кроме билинейного (гейзенберговского) обменного взаимодействие, также и биквадратичное обменное взаимодействие. Кроме того, будем рассматривать эти взаимодействия существенно анизотропными, т.е. гамильтониан такой системы можно представить в виде:

$$
\begin{aligned}
H= & -\frac{1}{2} \sum_{n \neq n^{\prime}}\left\{J_{n n^{\prime}}\left(S_{n}^{z} S_{n^{\prime}}^{z}+\Delta\left(S_{n}^{x} S_{n^{\prime}}^{x}+S_{n}^{y} S_{n^{\prime}}^{y}\right)\right)\right. \\
& +K_{n n^{\prime}}\left(O_{2 n}^{0} O_{2 n^{\prime}}^{0} / 3+\Delta_{1}\left(O_{2 n}^{z x} O_{2 n^{\prime}}^{z x}+O_{2 n}^{y z} O_{2 n^{\prime}}^{y z}\right)\right. \\
& \left.\left.+\Delta_{2}\left(O_{2 n}^{2} O_{2 n^{\prime}}^{2}+O_{2 n}^{x y} O_{2 n^{\prime}}^{x y}\right)\right)\right\},
\end{aligned}
$$

где $J_{n n}, K_{n n^{\prime}}-$ константы билинейного и биквадратичного обменных взаимодействий соответственно; $\Delta, \Delta_{1}, \Delta_{2}-$ параметры межионной анизотропии; $O_{j}^{i}(j \leq 2 S, i \leq j)$ - операторы Стивенса [16], связанные со спиновыми операторами следующим образом:

$$
\begin{gathered}
O_{2}^{0}=3\left(S^{z}\right)^{2}-S(S+1), \quad O_{2}^{2}=\frac{1}{2}\left[\left(S^{+}\right)^{2}+\left(S^{-}\right)^{2}\right] \\
O_{2}^{x y}=\frac{1}{2 i}\left[\left(S^{+}\right)^{2}-\left(S^{-}\right)^{2}\right] \\
O_{2}^{z x}=\frac{1}{2}\left[S^{z},\left(S^{+}+S^{-}\right)\right]_{+}, O_{2}^{y x}=\frac{1}{2 i}\left[S^{z},\left(S^{+}-S^{-}\right)\right]_{+}
\end{gathered}
$$

Такой выбор представления гамильтониана (1) связан с тем, что полный набор операторов (2) совместно с операторами $S^{x}, S^{y}, S^{z}$ являются генераторами группы $S U$ (3).

Будем рассматривать поведение системы в двух предельных случаях.

1. Все параметры межионной анизотропии положительны $\left(\Delta, \Delta_{1}, \Delta_{2}>0\right)$.

2. Все параметры межионной анизотропии отрицательны $\left(\Delta, \Delta_{1}, \Delta_{2}<0\right)$.

Как будет показано ниже, первый случай (с положительными параметрами межионной анизотропии) можно рассматривать как двухосный негейзенберговский магнетик, а второй случай (с отрицательными параметрами межионной анизотропии) представляет собой изингоподобный негейзенберговский магнетик. При этом, гамильтониан (1) описывает изотропный негейзенберговский магнетик, если все параметры межионной анизотропии равны единице.

Исследование свойств магнетика будем проводить в области низких температур $\left(T \ll T_{c}, T_{c}\right.$ - температура Кюри), поскольку именно в этом случае наиболее ярко проявляются квантовые свойства системы.

Рассмотрим вначале случай, когда все параметры межионной анизотропии положительны $\left(\Delta, \Delta_{1}, \Delta_{2}>0\right)$.
Выбором системы координат относительно кристаллографических осей [17] можно добиться, чтобы параметры порядка, характеризующие систему, были действительными, т.е. $\left\langle S^{y}\right\rangle=\left\langle O_{2}^{y z}\right\rangle=\left\langle O_{2}^{x y}\right\rangle=0$. Учет анизотропного обменного взаимодействия приводит к тому, что в общем случае магнитный момент направлен под углом $\theta$ к оси $O Z$. Унитарный поворот $U(\theta)=\prod_{n} \exp \left(i \theta S_{n}^{y}\right)$ позволяет перейти в собственную систему координат для каждого узла, в которой направление оси $O Z$ совпадает с направлением среднего спина. В результате этих преобразований гамильтониан (1) будет зависеть от угла $\theta$

$$
\tilde{H}(\theta) \rightarrow U(\theta) H U^{+}(\theta) .
$$

Выделяя в гамильтониане $\tilde{H}(\theta)$ средние поля, связанные как с векторным параметром порядка $\left(\left\langle S^{z}\right\rangle\right)$, так и с тензорным $\left(q_{2}^{t}=\left\langle O_{2}^{t}\right\rangle\right)$, получим одноузельный гамильтониан

$$
H_{0}(n)=-H_{z} S_{n}^{z}-H_{x} S_{n}^{x}-B_{2}^{0} O_{2 n}^{0}-B_{2}^{2} O_{2 n}^{2}-B_{2}^{z x} O_{2 n}^{z x},
$$

где $\quad H_{z}=J_{0}\langle S\rangle\left(\cos ^{2} \theta+\Delta \sin ^{2} \theta\right), \quad H_{x}=J_{0}\langle S\rangle \sin \theta$ $\times \cos \theta(1-\Delta), \quad$ коэффициенты $B_{2}^{0}, B_{2}^{2}, B_{2}^{z x}-$ пропорциональны $K_{0}$ и являются функциями квадрупольных параметров $q_{2}^{t}=\left\langle O_{2}^{t}\right\rangle \quad(t=0,2, z x) ; J_{0}=\sum_{n^{\prime}} J_{n n^{\prime}}$, $K_{0}=\sum_{n^{\prime}} K_{n n^{\prime}}$

Решая одноузельную задачу с гамильтонианом (3), найдем базис собственных

$$
\begin{gathered}
|\psi(1)\rangle=\cos \beta(\cos \alpha|1\rangle+\sin \alpha|-1\rangle)+\sin \beta|0\rangle, \\
|\psi(0)\rangle=-\sin \beta(\cos \alpha|1\rangle+\sin \alpha|-1\rangle)+\cos \beta|0\rangle, \\
|\psi(-1)\rangle=-\sin \alpha|1\rangle)+\cos \alpha|-1\rangle,
\end{gathered}
$$

в котором гамильтониан (3) становится диагональным. На базисе (4) построим операторы Хаббарда $X^{M^{\prime} M}=\left|\psi\left(M^{\prime}\right)\right\rangle\langle\psi(M)[18]$, из связи которых со спиновыми операторами определяется вид параметров порядка

$$
\begin{gathered}
\left\langle S^{z}\right\rangle=\cos 2 \alpha \cos ^{2} \beta, \quad q_{2}^{0}=\cos ^{2} \beta-2 \sin ^{2} \beta, \\
q_{2}^{2}=\sin 2 \alpha \cos ^{2} \beta, \quad q_{2}^{z x}=(\cos \alpha-\sin \alpha) \sin 2 \beta / \sqrt{2} .
\end{gathered}
$$

Кроме того, в результате унитарных преобразований возникает еще один параметр [19]

$$
\left\langle S^{x}\right\rangle=(\cos \alpha+\sin \alpha) \sin 2 \beta / \sqrt{2},
$$

который в собственной системе координат равен нулю. Из условия $\left\langle S^{x}\right\rangle=0$ следует, что $\beta=0$, поскольку, в случае $\alpha=-\pi / 4$ или $\beta=\pi / 2$ не только $\left\langle S^{x}\right\rangle=0$, но и $\left\langle S^{z}\right\rangle=0$, что приводит к потере решений для ферромагнитно упорядоченных состояний. С учетом этого параметры в собственной системе координат можно представить в виде

$$
\langle S\rangle=\cos 2 \alpha, \quad q_{2}^{0}=1, \quad q_{2}^{2}=\sin 2 \alpha, \quad q_{2}^{z x}=0 .
$$


В случае низких температур $(T \rightarrow 0)$ энергия основного состояния совпадает с плотностью свободной энергии. Анализ плотности свободной энергии

$$
\begin{aligned}
F & =\frac{1}{2}\left[J_{0}(1-\Delta) \cos ^{2} 2 \alpha+K_{0}\left(1-\Delta_{1}+\left(\Delta_{1}-\Delta_{2}\right) \sin 2 \alpha\right)\right. \\
& \times(1-\sin 2 \alpha)] \sin ^{2} \theta+\frac{K_{0}}{8}\left(4 \Delta_{1}-3-\Delta_{2}\right)(1-\sin 2 \alpha)^{2} \\
& \times \sin ^{4} \theta-\frac{1}{2}\left(J_{0}-K_{0} \Delta_{2}\right) \cos ^{2} 2 \alpha
\end{aligned}
$$

позволяет определить спиновые состояния, реализуемые в системе при различных соотношениях материальных параметров.

\section{3. „Двухосный“ негейзенберговский магнетик}

Хорошо известно, что в негейзенберговском изотропном магнетике ферромагнитная фаза устойчива при $J_{0}>K_{0}[1,20]$. Учет анизотропии обменного взаимодействия это условие скорректирует.

Очевидно, что в случае преобладающего билинейного обменного взаимодействия величина среднего спина практически не меняется $-\langle S\rangle \simeq 1$ (параметр $\alpha=0$ ). В зависимости от значения анизотропного параметра $\Delta$ изменяется направление вектора намагниченности и реализуются ферромагнитно упорядоченные фазы: легкоосная ферромагнитная фаза $\left(\Phi M_{\|}\right)$с направлением вектора магнитного момента вдоль оси $O Z$, устойчивая при $\Delta<1$, и легкоплоскостная ферромагнитная фаза $\left(\Phi M_{\perp}\right)$ с направлением вектора магнитного момента, ортогональным оси $O Z$, устойчивая при $\Delta>1$. Кроме того, возникает промежуточное состояние, в котором векторный параметр порядка (магнитный момент $\langle S\rangle$ ) является ненасыщенным. Это обстоятельство связано квантовой редукцией спина [9], т.е. модуль магнитного момента $\langle S\rangle$ может меняться от нуля до единицы, в зависимости от соотношения материальных констант

$$
\begin{gathered}
\langle S\rangle=\sqrt{1-\sin ^{2} 2 \alpha_{\perp}}, \\
\sin 2 \alpha_{\perp}=K_{0}\left(\Delta_{2}-1\right) /\left(4 J_{0} \Delta-K_{0}\left(3+\Delta_{2}\right)\right) .
\end{gathered}
$$

Тогда легкоплоскостная $\left(\Phi M_{\perp}\right)$ фаза при $K_{0} \neq 0$ трансформируется в квадрупольно-ферромагнитную $\left(K \Phi M_{\perp}\right)$ фазу, которая характеризуется тем, что в ней дипольный и квадрупольные параметры порядка могут изменяться от максимального до минимального значений, в зависимости от соотношения материальных констант. Так, при $\Delta_{2}=1$ намагниченность достигает насыщения и $\langle S\rangle=1$.

Кроме того, как следует из (6), при $4 \Delta_{1}-3-\Delta_{2}>0$ возможна реализация угловой квадрупольно ферромагнитной фазы $\left(K \Phi M_{L}\right)$ с $\langle S\rangle<1$, а направление вектора магнитного момента не имеет фиксированной ориентации, и в зависимости от соотношения между материальными константами значение угла $\theta$ может меняться от 0 до $\pi / 2$

$$
\begin{array}{r}
\sin 2 \alpha_{\angle}=\frac{K_{0} \sin ^{2} \theta\left(1-\Delta_{2}+\left(4 \Delta_{1}-3-\Delta_{2}\right) \cos ^{2} \theta\right)}{K_{0}\left(4 \Delta_{2}+3\left(1-\Delta_{2}\right) \sin ^{2} \theta+\left(4 \Delta_{1}-3-\Delta_{2}\right) \times\right.} \\
\left.\times \sin ^{2} \theta \cos ^{2} \theta\right)-4 J_{0}\left(\cos ^{2} \theta+\Delta \sin ^{2} \theta\right)
\end{array}
$$

Дополнительную информацию об областях существования соответствующих спиновых состояний и о типах фазовых переходов между ними могут дать исследования динамических свойств системы, т.е. спектров магнонов. Спектры элементарных возбуждений, определяющиеся полюсами функции Грина [21]

$$
G^{\lambda \lambda^{\prime}}\left(n, \tau ; n^{\prime}, \tau^{\prime}\right)=-\left\langle\hat{T} \tilde{X}_{n}^{\lambda}(\tau) \tilde{X}_{n^{\prime}}^{\lambda^{\prime}}\left(\tau^{\prime}\right)\right\rangle,
$$

где $\tilde{X}_{n}^{\lambda}(\tau)=\exp (\hat{H} \tau) X_{n}^{\lambda} \exp (-\hat{H} / \tau)$ - оператор Хаббарда в гейзенберговском представлении, $\hat{T}$ - оператор Вика, $\lambda$ - корневые вектора, определяющиеся алгеброй операторов Хаббарда [18]. Мы не будем здесь останавливаться на выводе дисперсионного уравнения, поскольку этот вопрос подробно изложен в [18,22]. Дисперсионное уравнение справедливо при произвольном соотношении материальных констант и в широком температурном интервале существования магнитного упорядочения (исключая флуктуационную область). Решения дисперсионного уравнения определяют спектры элементарных возбуждений в различных фазах.

Спектры магнонов в легкоосной $\Phi M_{\|}$и легкоплоскостной $K \Phi M_{\perp}$ фазах имеют вид соответственно

$$
\begin{gathered}
\varepsilon_{1}(k)=2\left(J_{0}-K_{k} \Delta_{2}\right) ; \\
\varepsilon_{1}(k)=2 \sqrt{\begin{array}{c}
\left(J_{0} \Delta-K_{k} \Delta_{1}\right)\left\{\left(J_{0}-J_{k}\right) \Delta+\right. \\
\left.+\left[J_{k} \Delta-K_{k}\left(3+\Delta_{2}\right) / 4\right]\left(1-\sin ^{2} 2 \alpha_{\perp}\right)\right\}
\end{array}} \\
\Omega_{1}=\left(J_{0}-J_{k}\right) \Delta\left(1-\sin 2 \alpha_{\perp}\right)+\left(K_{0}-K_{k}\right) \Delta_{2}\left(1+\sin 2 \alpha_{\perp}\right), \\
\Omega_{2}=J_{k}(\Delta-1)-K_{k}\left(\Delta_{2}-\Delta_{1}\right) \\
+\sin 2 \alpha_{\perp}\left(K_{k}\left(\Delta_{2}+\Delta_{1}\right)-J_{k}(\Delta+1)\right) .
\end{gathered}
$$

Исследуем поведение спектров возбуждения в наиболее интересных случаях. Пусть $\Delta_{1}>\left(3+\Delta_{2}\right) / 4$. Фазовые переходы ,легкоосная-угловая“ и ,легкоплоскостная-угловая“ фазы являются ориентационными фазовыми переходами второго рода. При приближении к линии фазового перехода „угловая-легкоосная“ модуль магнитного момента стремится к значению насыщения $\langle S\rangle=1$. На линии фазового перехода $J_{0}(1-\Delta)=K_{0}\left(\Delta_{1}-1\right)$ щель в спектре (9.2) обращается 
в ноль. Как следует из выражений (7) и (8) в легкоплоскостной $K \Phi M_{\perp}$ и угловой $K \Phi M_{\angle}$ фазах $\langle S\rangle \neq 1$. На линии фазового перехода „угловая-легкоплоскостная“ фазы

$J_{0}[1-\Delta+(1+\Delta) \sin 2 \alpha]=K_{0}\left[\Delta_{2}-\Delta_{1}+\sin 2 \alpha_{\perp}\left(\Delta_{2}+\Delta_{1}\right)\right]$,

значения намагниченностей сравниваются, т. е.

$$
\sin 2 \alpha_{\perp}=\sin 2 \alpha_{\angle} \quad \text { при } \quad \theta=\pi / 2 .
$$

На этой же линии обращается в ноль щель в спектре (10.2). В длинноволновом пределе $k \rightarrow 0$ оба спектра (9.2) и (10.2) на линиях фазовых переходов в угловую фазу имеют квадратичный закон дисперсии. Такое поведение спектров соответствует ориентационным фазовым переходам второго рода.

Если параметры межионной анизотропии соотносятся как $\Delta_{1}<\left(3+\Delta_{2}\right) / 4$, то в этом случае угловая фаза становится неустойчивой. Фазовый переход из легкоосной фазы происходит непосредственно в легкоплоскостную фазу и не является чисто ориентационным, так как на линии фазового перехода легкоосная-легкоплоскостная фазы вместе с поворотом вектора намагниченности на угол $\pi / 2$ скачком изменяется значение модуля вектора намагниченности с $\langle S\rangle=1$ в легкоосной фазе до $\langle S\rangle<1$ в легкоплоскостной фазе (см. выражение (7)). Линия фазового перехода определяется из равенства свободных энергий в легкоосной и легкоплоскостной фазах и имеет вид

$$
4 J_{0}(1-\Delta)=K_{0}\left(1-\Delta_{2}\right)\left(1+\sin 2 \alpha_{\perp}\right) .
$$

В окрестности линии фазового перехода „легкоосная-легкоплоскостная" фазы размягчаются ветви (9.2) и (10.2). Обращение в ноль щелей в спектрах определяют линии потери устойчивости спектров: $J_{0}(1-\Delta)=K_{0}\left(\Delta_{1}-1\right) \quad$ и $\quad J_{0}[1-\Delta+(1+\Delta) \sin 2 \alpha]=$ $=K_{0}\left[\Delta_{2}-\Delta_{1}+\sin 2 \alpha\left(\Delta_{2}+\Delta_{1}\right)\right]$. Эти линии определяют границы области сосуществования рассматриваемых здесь фаз. Такое поведение спектров характерно для фазовых переходов первого рода.

Рассмотрим теперь противоположную ситуацию, а именно, константа биквадратичного обмена существенно больше константы билинейного обменного взаимодействия $\left(K_{0}>J_{0}\right)$. Как было показано в целом ряде работ (см., например, $[2,20,23,24])$, в изотропном негейзенберговском магнетике с $S=1$ в случае большого биквадратичного обменного взаимодействия ферромагнитное упорядочение становится энергетически невыгодным. Дипольный параметр порядка становится равным нулю, и система характеризуется высшими, по компонентам спинового оператора, мультипольными параметрами порядка. Для исследуемой нами модели нематические фазы, как следует из (5), это состояние характеризуется квадрупольными параметрами порядка $q_{2}^{0}$ и $q_{2}^{2}$, являющимися диагональными компонентами симметричного тензора второго ранга $q_{2}^{i j}=\left\langle S^{i} S^{j}+S^{j} S^{i}\right\rangle / 2$.

Анализ плотности свободной энергии (5), при соотношении обменных интегралов $K_{0}>J_{0}$, показывает, что в этом случае могут реализоваться следующие фазовые состояния с $\langle S\rangle=0$ :

- нематическая плоскостная $N_{1}$ фаза при $\theta=0$ и $\alpha=-\pi / 4$;

- нематическая осевая $N_{2}$ фаза при $\theta=\pi / 2$ и $\alpha=-\pi / 4$

- угловая нематическая фаза $N_{\angle}$ при $\cos 2 \theta=\left(1-\Delta_{2}\right)$ $\times\left(3+\Delta_{2}-4 \Delta_{1}\right)$ и $\alpha=-\pi / 4$.

Нематические состояния характеризуются параметрами порядка, которые являются компонентами симметричного тензора второго ранга; геометрическим образом такого тензора (с положительными компонентами) в трехмерном пространстве является одноосный эллипсоид. Его ориентацию можно задать единичным вектором n, ортогональным плоскости квадрупольного эллипсоида (в данном случае диска), и в этом проявляется аналогия с молекулярным нематиком, в котором ориентация молекул в пространстве характеризуется единичным вектором-директором [25]. Также как и в молекулярном нематике, в спиновом нематике направления векторов $\mathbf{n}$ и $-\mathbf{n}$ являются неразличимыми и величина $q_{2}^{i j}$ является квантовым аналогом параметра порядка Де Жена, который вводится для обычных нематических жидких кристаллов [25].

Из анализа свободной энергии (6) следует, что основное состояние нематических фаз связанно с направлением вектора-директора $\mathbf{n}$, определяется основной функцией $\psi=(|1\rangle-|-1\rangle) / \sqrt{2}$. В собственной системе координат вектор-директор $\mathbf{n}$ параллелен оси $O X$, так как „диск к квадрупольного момента имеет отличные от нуля компоненты $\left\langle\left(S^{y}\right)^{2}\right\rangle=\left\langle\left(S^{z}\right)^{2}\right\rangle=1$. Угол $\theta$ определяет отклонение вектора $\mathbf{n}$ от оси $O X$ лабораторной системы координат. Для определения вида собственных функций в лабораторной системе координат необходимо провести обратное унитарное преобразование $U(-\theta)=\exp \left(-i \theta S^{y}\right)$. Действуя оператором $U(-\theta)=\exp \left(-i \theta S^{y}\right)$ при $\theta=\pi / 2$ на функцию $\psi=(|1\rangle-|-1\rangle) / \sqrt{2}$ определяем вид функции основного состояния $\psi=|0\rangle$ нематической фазы $N_{2}$ в лабораторной системе координат. Параметры порядка, характеризующие $N_{2}$ фазу: $q_{2}^{0}=-2, q_{2}^{2}=0$. В нематической $N_{1}$-фазе функция основного состояния: $\psi=(|1\rangle-|-1\rangle) / \sqrt{2}$ и параметры порядка: $q_{2}^{0}=1$, $q_{2}^{2}=-1$. Необходимо отметить, что в изотропном случае состояния $N_{1}$ и $N_{2}$ являются вырожденными, т.е. их энергии совпадают, а соответствующие им волновые функции переходят друг в друга непрерывным поворотом. Однако для обменно анизотроного магнетика ситуация иная: как следует из (6), различным состояниям соответствует различная свободная энергия и, следовательно, обменная анизотропия снимает вырождение этих спиновых состояний. Кроме того, необходимо отметить, что спиновый нематик $N_{2}$ по своим симметрийным свойствам напоминает квадрупольное состояние, возникающее в магнетике с большой легкоплоскостной одноионной анизотропией [26,27].

Одним из интересных моментов в изучении данной модели является возникновение угловой нематической 
фазы [28]. Функция основного состояния данной фазы в лабораторной системе координат

$$
\psi=\cos \theta(|1\rangle-|-1\rangle) / \sqrt{2}+\sin \theta|0\rangle
$$

представляет суперпозицию двух векторов нематических состояний $(|1\rangle-|-1\rangle) / \sqrt{2}$ и $|0\rangle$. Как видно из фазовой диаграммы в плоскости $\left(\Delta_{1}, \Delta_{2}\right)$, при фиксированных параметрах $\Delta$ и $J_{0} / K_{0}<1$ (рис. 1 ) нематическая угловая фаза $N_{\angle}$ переходит при $\Delta_{2}<1$ и $\Delta_{1}=1$ в осевую $N_{2}$ фазу, а при $\Delta_{2}<1$ и $\Delta_{1}=\left(1+\Delta_{2}\right) / 2$ в плоскостную нематическую $N_{1}$ фазу.

Особо следует подчеркнуть, что необходимое условие устойчивости угловой нематической фазы, возникающее из анализа свободной энергии (6), аналогично условию существования угловой ферромагнитной фазы, а именно $\Delta_{1}>\left(3+\Delta_{2}\right) / 4$. Для понимания особенностей формирования спиновых конфигураций необходимо исследовать динамику спиновых возбуждений. Приведем решения дисперсионного уравнения в нематических фазах.

Спектры элементарных возбуждений в угловой $\left(N_{\angle}\right)$ фазе имеют вид

$$
\begin{gathered}
\varepsilon_{1}(k)=2 \sqrt{\begin{array}{c}
\left(K_{0} \Delta_{1}-J_{k} \Delta\right)\left(\left(K_{0}-K_{k}\right) \Delta_{1}+\right. \\
\left.+K_{k} \frac{4 \Delta_{1}-3-\Delta_{2}}{4}\left(1-\cos ^{2} 2 \theta\right)\right)
\end{array}} \\
\varepsilon_{2}(k)=\sqrt{\tilde{\Omega}_{1}\left(\tilde{\Omega}_{1}+\tilde{\Omega}_{2}\right)} \\
\tilde{\Omega}_{1}=\left(K_{0}-K_{k}\right)\left(\Delta_{2}-\Delta_{1}+\cos 2 \theta\left(\Delta_{2}-\Delta_{1}\right)\right) \\
\tilde{\Omega}_{2}=K_{k}\left(\Delta_{2}-\Delta_{1}\right)-J_{k}(\Delta+1) \\
+\cos 2 \theta\left(K_{k}\left(\Delta_{2}-\Delta_{1}\right)+J_{k}(\Delta-1)\right)
\end{gathered}
$$

а в $N_{1}$ фазе спектры можно представить в виде:

$$
\begin{gathered}
\varepsilon_{1}(k)=2 \sqrt{\left(K_{0} \Delta_{2}-J_{k}\right)\left(K_{0}-K_{k}\right) \Delta_{2}} \\
\varepsilon_{2}(k)=\sqrt{\left(K_{0}\left(1+\Delta_{2}\right)-2 J_{k} \Delta\right)\left(K_{0}\left(1+\Delta_{2}\right)-2 K_{k} \Delta_{1}\right)} .
\end{gathered}
$$

В $N_{2}$ фазе, вследствие вырождения возбужденных одноионных состояний, спектры магнонов совпадают

$$
\varepsilon_{1}(k)=\varepsilon_{2}(k)=2 \sqrt{\left(K_{0}-J_{k} \Delta\right)\left(K_{0}-K_{k} \Delta_{1}\right)} .
$$

Необходимо отметить, что при переходе к изотропному случаю $\left(\Delta=\Delta_{1}=\Delta_{2}=1, \theta=0\right)$ все три фазы $N_{1}, N_{2}$ и $N_{\angle}$ становятся неразличимы, их основное состояние $\psi=(|1\rangle+|-1\rangle) / \sqrt{2}$, а спектры принимают стандартный вид $[17,23,29]$

$$
\varepsilon_{1}(k)=\varepsilon_{2}(k)=2 \sqrt{\left(K_{0}-J_{k}\right)\left(K_{0}-K_{k}\right)} .
$$

Таким образом, обменная анизотропия снимает „вырождение“ нематических состояний, которое наблюдается в изотропном негейзенберговском магнетике.

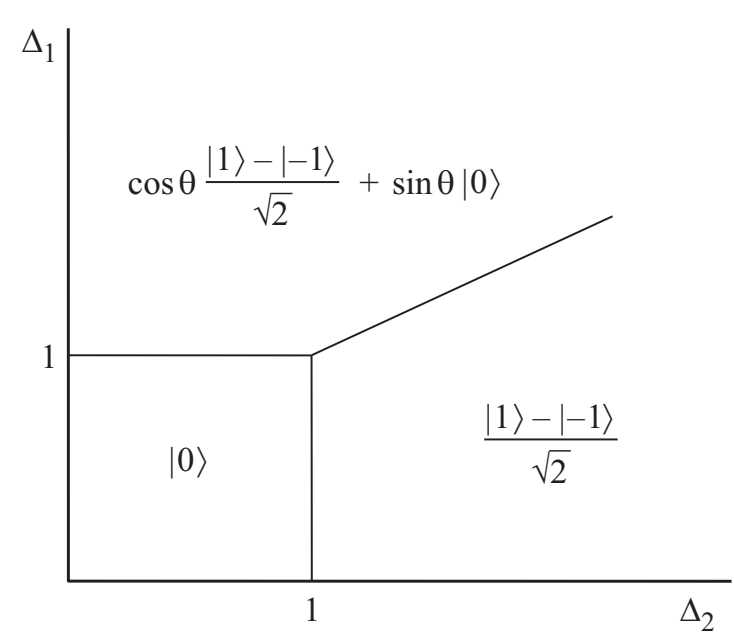

Рис. 1. Фазовая диаграмма обменно анизотропного негейзенберговского магнетика на плоскости при $\left(\Delta_{1}, \Delta_{2}\right)$ при $J_{0} / K_{0}<1$.

Фазовые переходы между нематическими фазами являются ориентационными и происходят по параметрам анизотропии биквадратичного обменного взаимодействия (рис. 1). Поскольку в нематических фазах магнитный момент (на узле) равен нулю, то понятие „переориентации“ связано с поворотом векторадиректора n. В случае $\Delta_{1}>\left(3+\Delta_{2}\right) / 4$ фазовые переходы между $N_{1}-N_{\angle}$ и $N_{\angle}-N_{2}$ фазами являются ориентационными фазовыми переходами второго рода. На линии $\Delta_{1}=\left(1+\Delta_{2}\right) / 2$ фазового перехода $N_{1}-N_{\angle}$ щели в спектрах (11.1) и (12.2) обращаются в ноль, и на этой линии спектры становятся линейными по волновому вектору $\mathbf{k}$ в длинноволновом пределе. Аналогично на линии фазового перехода $N_{2}-N_{\angle} \Delta_{1}=1$ при $k \rightarrow 0$ щели в ветвях (11.1) и (13) обращаются в ноль, а спектры также становятся линейными по волновому вектору.

Если $\Delta_{1}<\left(3+\Delta_{2}\right) / 4$, то фазовый переход между $N_{1}$ и $N_{2}$ фазами происходит скачком на линии $\Delta_{2}=1$. Такой фазовый переход является ориентационным переходом первого рода - направление вектора $\mathbf{n}$ меняется скачком, и не сопровождается размягчением спектров магнонов на линии фазового перехода $\Delta_{2}=1$.

Таким образом, имеется существенное отличие в поведении негейзенберговских магнетиков в окрестности ориентационных фазовых переходов по материальным параметрам для изотропного и анизотропного обменных взаимодействий. Как показало исследование модели негейзенберговского магнетика с изотропным обменным взаимодействием [30], ориентационные фазовые переходы являются вырожденными переходами первого рода: одновременно на границе фаз скачком меняется симметрия фаз и имеется размягчение спектров элементарных возбуждений. В рассматриваемой модели ориентационные фазовые переходы между ферромагнитными и нематическими фазами происходят при измении констант анизотропного обменного взаимодей- 


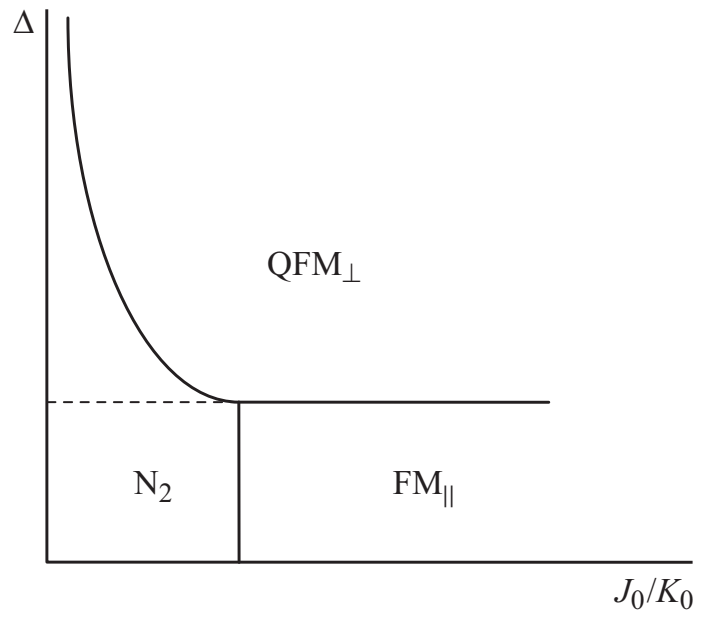

Рис. 2. Фазовая диаграмма обменно анизотропного негейзенберговского магнетика на плоскости $\left(\Delta, J_{0} / K_{0}\right)$ в случае реализации ферромагнитных и нематических фаз при $\Delta_{1}<1$, $\Delta_{2}<1$.

ствия, причем могут быть как первого, так и второго рода.

Рассмотрим поведение системы на границах ферромагнитных и нематических фаз. Фазовые переходы такого типа связаны с обращением намагниченности в ноль на одном узле - т. е. эффектом квантового сокращения спина $[17,19]$. В целом ряде работ, например [30,31], показано, что одноионная магнитная анизотропия и обменное биквадратичное взаимодействие усиливают друг друга: биквадратичное обменное взаимодействие и одноионная анизотропия приводят к неэквидистантному расталкиванию уровней энергии магнитного иона. Однако биквадратичный обмен стремится установить нематический порядок в целом с резкой сменой ферромагнитного порядка с $\langle S\rangle=1$ на нематический $\langle S\rangle=0[1,5]$. При этом, рост константы одноионной анизотропии приводит к плавному уменьшению среднего спина на узле и реализации промежуточного квадрупольно-ферромагнитного упорядочения [31,32]. В данной модели квадрупольноферромагнитные фазы реализуются благодаря анизотропному обменному взаимодействию.

Поскольку фазовые состояния исследуемой системы зависят от большого числа параметров, невозможно графически отобразить фазовый портрет системы. Но мы можем построить фазовую диаграмму системы на различных плоскостях.

Вначале рассмотрим случай $\Delta_{2}<1, \Delta_{1}<1$ (рис. 2). При таком соотношении параметров биквадратичной анизотропии и в зависимости от соотношения между $\Delta$ и $J_{0} / K_{0}$ в системе реализуются $\Phi M_{\|}, K \Phi M_{\perp}$ и $N_{2}$ фазы. Поведение магнетика в окрестности ориентационного фазового перехода $\Phi M_{\|}-K \Phi M_{\perp}$ было исследовано выше.

Рассмотрим теперь поведение системы в окрестности фазовых переходов $\Phi M_{\|}-N_{2}$ и $K \Phi M_{\perp}-N_{2}$. Из равенства свободных энергий в $\Phi M_{\|}$и $N_{2}$ фазах удается определить линию фазового перехода $\Phi M_{\|}-N_{2}: J_{0}=K_{0}$. При этом, как следует из (9.1) и (13), энергетические щели в спектрах магнонов соответствующих фаз обращаются в ноль не на линии $J_{0}=K_{0}$, а на линиях $J_{0}=K_{0} \Delta_{2}$ в области $J_{0} / K_{0}<1$ и на линии $J_{0} \Delta=K_{0}$ в области $J_{0} / K_{0}>1$. Эти линии являются линиями потери устойчивости соответствующих фаз и определяют область сосуществования $\Phi M_{\|}$и $N_{2}$ фаз. Таким образом, фазовый переход $\Phi M_{\|}-N_{2}$ является фазовым переходом первого рода, в результате которого скачком меняется модуль намагниченности с единицы до нуля.

Линия фазового перехода $K \Phi M_{\perp}-N_{2}$ определяется выражением $J_{0} \Delta=K_{0}$. На этой же линии обращаются в ноль щели спектров магнонов (10.1) и (13). Данный фазовый переход является фазовым переходом второго рода: при приближении к линии фазового перехода из квадрупольно-ферромагнитной фазы $K \Phi M_{\perp}$ намагниченность непрерывно уменьшается и на линии $J_{0} \Delta=K_{0}$ обращается в ноль.

Рассмотрим еще одну ситуацию, когда параметры межионной анизотропии связаны соотношениями $\Delta_{2}>1$ и $\Delta_{1}<\left(1+\Delta_{2}\right) / 2$ (рис. 3 ). В этом случае в системе в зависимости от соотношений $\Delta$ и $J_{0} / K_{0}$ реализуются $\Phi M_{\|}, K \Phi M_{\perp}$ и $N_{1}$ фазы.

Линия фазового перехода $\Phi M_{\|}-N_{1}$ фазы $J_{0}=K_{0} \Delta_{2}$ определяется из равенства свободных энергий. На этой линии обращаются в ноль щели ветвей возбуждения (9.1) и (12.1). Переход между фазами сопровождается скачком намагниченности. Данный фазовый переход является вырожденным фазовым переходом первого рода: симметрия фаз меняется скачком, но тем не менее щель в спектрах обращается на линии фазового перехода в ноль. Линия фазового перехода $K \Phi M_{\perp}-N_{1}$ определяется выражением $2 J_{0} \Delta=K_{0}\left(1+\Delta_{2}\right)$. На этой же линии обращаются в ноль щели спектров (10.1) и (12.2). Необходимо отметить следующую особенность: на линии $J_{0}=K_{0} \Delta_{2}$ в длинноволновом пределе ветви возбуждения (9.1) и (12.1) имеют квадратичный закон дисперсии

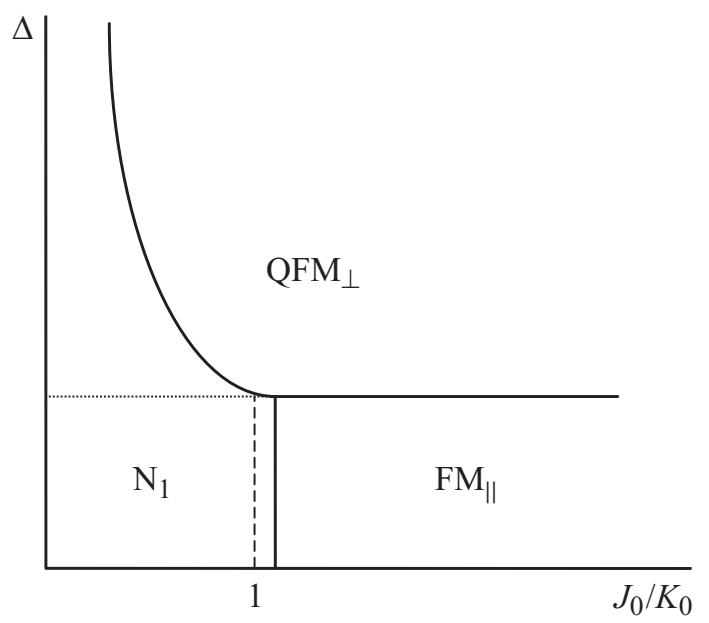

Рис. 3. Фазовая диаграмма обменно анизотропного негейзенберговского магнетика на плоскости $\left(\Delta, J_{0} / K_{0}\right)$ в случае реализации ферромагнитных и нематических фаз при $\Delta_{2}>1$, $\Delta_{1}<\left(1+\Delta_{2}\right) / 2$. 


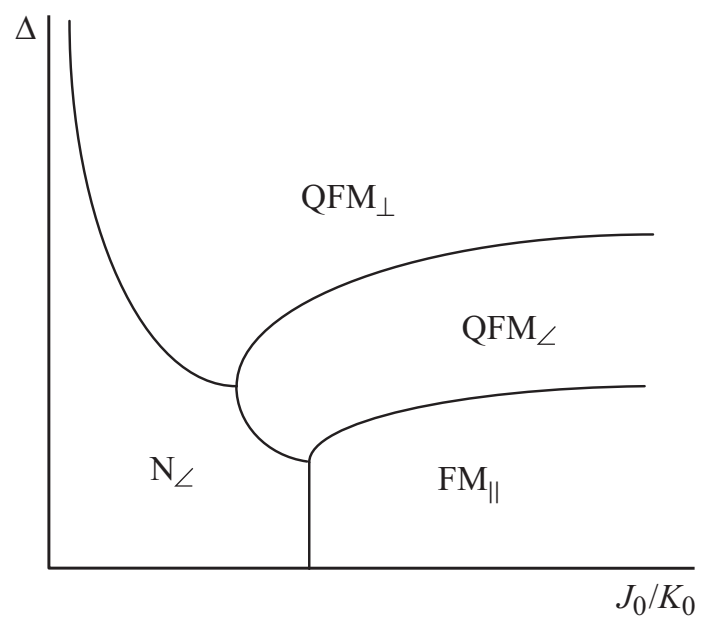

Рис. 4. Фазовая диаграмма обменно анизотропного негейзенберговского магнетика на плоскости $\left(\Delta, J_{0} / K_{0}\right)$ в случае реализации ферромагнитных и нематических фаз при $\Delta_{1}>1$, $\Delta_{1}>\left(3+\Delta_{2}\right) / 4$.

по волновому вектору; на линии $2 J_{0} \Delta=K_{0}\left(1+\Delta_{2}\right)$ спектры (10.1) и (12.2) будут линейны по волновому вектору $\mathbf{k}$.

Рассмотрим теперь фазовую диаграмму на плоскости $\left(\Delta, J_{0} / K_{0}\right)$ при $\Delta_{1}>1$ и $\Delta_{1}>\left(3+\Delta_{2}\right) / 4$ (рис. 4$)$. При таком соотношении параметров межионной обменной анизотропии, в зависимости от соотношений $\Delta$ и $J_{0} / K_{0}$, возможна реализация фаз $\Phi M_{\|}, K \Phi M_{\perp}, K \Phi M_{\angle}$ и $N_{\angle}$. Анализ спектров ферромагнитных фаз в окрестности линий ориентационных фазовых переходов был проведен выше.

Линия фазового перехода $\Phi M_{\|}-N_{\angle}$ определяется равенством свободных энергий

$$
J_{0}=K_{0} \Delta_{1}-\left(K_{0} / 4\right)\left(1-\Delta_{2}\right)(1+\cos 2 \theta),
$$

где $\cos 2 \theta=\left(1-\Delta_{2}\right) /\left(3+\Delta_{2}-4 \Delta_{1}\right)$. В окрестности линии фазового перехода (14) образуется область метастабильности, границы которой определяются обращением щелей в ноль в ветвях возбуждения (9.1), (11.2). Фазовый переход сопровождается скачкообразным изменением намагниченности.

Линия фазового перехода $K \Phi M_{\perp}-N_{\angle}$ фазы определяется выражением $J_{0} \Delta=K_{0} \Delta_{1}$. Приближаясь к этой линии из $K \Phi M_{\perp}$ фазы, намагниченность непрерывным образом стремится к нулю, а из $N_{\angle}$ фазы угол $\theta$ стремится к $\pi / 2$ и на линии фазового перехода принимают свои предельные значения. Таким образом, фазовый переход $K \Phi M_{\perp}-N_{\angle-}$ фазы является фазовым переходом второго рода, сопровождающийся непрерывным изменением намагниченности. Кроме того, на линии фазового перехода обращаются в ноль щели спектров (10.1), (11.1). На самой линии спектры при $k \rightarrow 0$ становятся квадратичными по волновому вектору.

Тот же тип фазового перехода $K \Phi M_{\angle}-N_{\angle-}$ фазы. При приближении к линии фазового перехода из $K \Phi M_{\angle-\text { фазы }}$ намагниченность стремится к нулю. На линии фазового перехода

$$
2\left(J_{0}-K_{0} \Delta_{2}\right)\left(1-\Delta_{1}\right)+\left(J_{0} \Delta-K_{0} \Delta_{1}\right)\left(1+\Delta_{2}-2 \Delta_{1}\right)=0
$$

параметр $\alpha=-\pi / 4$, соответственно намагниченность равна нулю, а угол $\theta$, как следует из выражений (7) при $\alpha=-\pi / 4$, определяется

$$
\cos 2 \theta_{\mid \alpha=-\pi / 4}=\left(1-\Delta_{2}\right) /\left(3+\Delta_{2}-4 \Delta_{1}\right)
$$

и совпадает с выражением, определяющим угол $\theta$ в

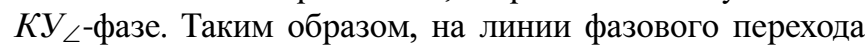
происходит непрерывное изменение модуля намагниченности. Щель в спектре (11.2) обращается в ноль, и спектр становится квадратичным по волновому вектору.

\section{4. „Изингоподобный“ негейзенберговский магнетик}

Рассмотрим теперь обменно анизотропный негейзенерговский магнетик со спином магнитного иона $S=1$ и отрицательными значениями параметров межионной анизотропии. Как будет показано ниже, такую систему можно рассматривать как негейзенберговский изингоподобный магнетик. Гамильтониан такой системы имеет вид, аналогичный (1) с заменой параметров межионной анизотропии $\Delta \rightarrow-\Delta, \Delta_{1} \rightarrow-\Delta_{1}, \Delta_{2} \rightarrow-\Delta_{2}$

$$
\begin{aligned}
H_{\text {int }}= & -\frac{1}{2} \sum_{n \neq n^{\prime}}\left\{J_{n n^{\prime}}\left(S_{n^{\prime}}^{z} S_{n^{\prime}}^{z}-\Delta\left(S_{n^{x}}^{x} S_{n^{\prime}}^{x}+S_{n}^{y} S_{n^{\prime}}^{y}\right)\right)\right. \\
& -K_{n n^{\prime}}\left(O_{2 n}^{0} O_{2 n^{\prime}}^{0} / 3-\Delta_{1}\left(O_{2 n}^{z x} O_{2 n^{\prime}}^{z x}+O_{2 n}^{y z} O_{2 n^{\prime}}^{y z}\right)\right. \\
& \left.\left.-\Delta_{2}\left(O_{2 n}^{2} O_{2 n^{\prime}}^{2}+O_{2 n}^{x y} O_{2 n^{\prime}}^{x y}\right)\right)\right\} .
\end{aligned}
$$

Все обозначения в (15) аналогичны введенным ранее. Отметим, что в предельном случае $\Delta, \Delta_{1}, \Delta_{2} \rightarrow-1$ гамильтониан (15) описывает изотропный негезенберговский магнетик. Очевидно, что знаки параметров межионной анизотропии $\Delta, \Delta_{1}, \Delta_{2}$ являются существенными при формировании фазовой картины магнетика.

Как и в предыдущем случае исследуем поведение системы в области низких температур при $T \ll T_{c}\left(T_{c}-\right.$ температура Кюри).

Переходя в (15) в собственную систему координат, связанную с осью квантования $O Z$, и средние поля, связанные с упорядочением магнитного момента, а также квадрупольных моментов, получим одноузельный гамильтониан, который имеет вид аналогично (3):

$$
H_{0}(n)=-H_{z} S_{n}^{z}-H_{x} S_{n}^{x}-B_{2}^{0} O_{2 n}^{0}-B_{2}^{2} O_{2 n}^{2}-B_{2}^{z x} O_{2 n}^{z x},
$$

где

$$
\begin{gathered}
H_{z}=J_{0}\langle S\rangle\left(\cos ^{2} \theta-\Delta \sin ^{2} \theta\right), H_{x}=J_{0}\langle S\rangle \sin \theta \cos \theta(1+\Delta), \\
B_{2}^{0}=K_{0}\left(A q_{2}^{2}+D q_{2}^{2}+H q_{2}^{x z}\right), B_{2}^{2}=K_{0}\left(D q_{2}^{2}+B q_{2}^{2}+G q_{2}^{x z}\right), \\
B_{2}^{x z}=K_{0}\left(H q_{2}^{2}+G q_{2}^{2}+C q_{2}^{x z}\right) .
\end{gathered}
$$


Амплитуды $A, B, C, D, G, H$ являются громоздкими функциями угла $\theta$ и параметров межионной анизотропии $\Delta_{1}, \Delta_{2}$.

Используя полученные ранее результаты, мы можем получить собственные функции и собственные значения гамильтониана (16), что позволяет определить плотность свободной энергии (при $T \rightarrow 0$ ) и параметры порядка как функции параметров унитарных преобразований и материальных параметров системы

$$
\begin{aligned}
F= & -\frac{1}{2} J_{0} \cos ^{2} 2 \alpha\left(\cos ^{2} \theta-\Delta \sin ^{2} \theta\right) \\
& +K_{0}\left\{-\frac{1}{2} A-D \sin 2 \alpha-\frac{1}{2} B \sin ^{2} 2 \alpha\right\},
\end{aligned}
$$

$$
\langle S\rangle=\cos 2 \alpha, \quad q_{2}^{0}=1, \quad q_{2}^{2}=\sin 2 \alpha, \quad q_{2}^{z x}=0 .
$$

Анализ плотности свободной энергии на экстремум по параметрам $\alpha$ и $\theta$ позволяет сделать предположение о возможности реализации однородных фазовых состояний в системе. Анализ энергетических уровней магнитного иона показывает, что основному состоянию системы соответствует $E_{1}$. Это позволяет внести дополнительный критерий реализации различных фазовых состояний. Из условия, что $E_{1}-$ нижайший уровень системы, разность $E_{-11}=E_{-1}-E_{1}$ должна быть положительной, так же как и $E_{01}=E_{0}-E_{1}$. Таким образом, сформулировано дополнительное условие устойчивости фаз.

$$
E_{01}=E_{0}-E_{1}>0, \quad E_{-11}=E_{-1}-E_{1}>0 .
$$

Остановимся более подробно на изучении системы при $J_{0}>K_{0}$. Анализ плотности свободной энергии (17) на экстремум показывает, что при $\langle S\rangle \neq 0$ возможно существование тех же ферромагнитных фазовых состояний, рассмотренных в предыдущем разделе: легкоосной ферромагнитной фазы $\left(\Phi M_{\|}\right)$, легкоплоскостной ферромагнитной фазы $\left(\Phi M_{\perp}\right)$ и квадрупольно ферромагнитной фазы $\left(K \Phi M_{\angle}\right)$. Однако более точный анализ плотности свободной энергии на минимум $\left(\frac{\partial^{2} F}{\partial \theta^{2}}>0, \frac{\partial^{2} F}{\partial \alpha^{2}}>0\right)$ и критерия (18) показывает, что кроме состояния $\Phi M_{\|}$ при $J_{0}>K_{0}$ остальные состояния не соответствуют минимуму термодинамического потенциала и становятся энергетически не выгодными.

Таким образом, при $J_{0}>K_{0}$ в системе реализуется лишь $\Phi M_{\|}$фаза $(\theta=0, \alpha=0)$ с вектором магнитного момента, параллельным оси $O Z$. Дополнительную информацию об области устойчивости однородных фаз можно получить из анализа спектров элементарных возбуждений. Процедура получения спектров магнонов подробно описана в работах $[12,18,22]$. Поэтому сразу приведем явный вид спектров магнонов в $\Phi M_{\|}$фазе

$$
\begin{gathered}
\varepsilon_{1}(k)=2\left(J_{0}+K_{0} \Delta_{2}\right), \\
\varepsilon_{2}(k)=J_{0}+J_{0} \Delta+K_{0}+K_{0} \Delta_{1} .
\end{gathered}
$$

Легко видеть, что спектры (19.1) и (19.2) являются бездисперсионными, а их энергетические щели не обращаются в ноль ни при каких значениях материальных параметров. Следовательно, спектры элементарных возбуждений становятся бездисперсионными и напоминают спектры модели Изинга [33]. На это же обстоятельство указывает вид гамильтониана (15), в котором слагаемое $-\frac{1}{2} \sum_{n \neq n^{\prime}}\left(S_{n}^{z} S_{n^{\prime}}^{z}-\Delta\left(S_{n}^{x} S_{n^{\prime}}^{x}+S_{n}^{y} S_{n^{\prime}}^{y}\right)\right)$ соответствует межионной анизотропии типа „легкая ось“. Все слагаемые, содержащие параметры межионной анизотропии в гамильтониане (15), имеют ту же геометрию, что и изингоподобный гамильтониан. Это означает, что спектры элементарных возбуждений в $\Phi M_{\|}$фазе не размягчаются ни при каких значениях параметров межионной анизотропии и, следовательно, фазовый переход из $\Phi M_{\|}$фазы не будет являться переориентационным.

Рассмотрим теперь противоположный случай, когда биквадратичное обменное взаимодействие превышает билинейное $\left(J_{0}<K_{0}\right)$. В этом случае ферромагнитный порядок становится энергетически невыгодным, и в системе реализуется нематическое упорядочение, определяемое квадрупольными параметрами порядка. Необходимо отметить, что нематический порядок не является парамагнитным, поскольку диагональные компоненты тензора квадрупольных моментов не равны друг другу [34].

Анализ плотности свободной энергии (15) показывает, что нематическое состояние реализуется при $\alpha=-\pi / 4$, а стабильным нематическим состоянием будет являться состояние $N_{2}$ (при $\left.\theta=\pi / 2\right)$ с волновой функцией основного состояния $|\psi\rangle=|0\rangle$. При этом угол $\theta$ уже определяет не направление магнитного момента, поскольку $\langle S\rangle=0$, а ориентацию вектора-директора. А нематические состояния $N_{1}$ с $\theta=0$ и $N_{\angle}$ становятся энергетически не выгодными.

В $N_{2}$ фазе, вследствие вырождения возбужденных одноионных энергетических состояний магнитного иона, спектры магнонов совпадают

$$
\varepsilon_{1}(k)=\varepsilon_{2}(k)=2 \sqrt{\left(K_{0}+J_{0} \Delta\right) K_{0}\left(1+\Delta_{1}\right)} .
$$

Так же как и в $\Phi M_{\|}$фазе, спектр (20) является бездесперсионным, и его анализ показывает, что он не обращается в ноль ни при каких значениях материальных параметров. Это обстоятельство свидетельствует о том, что данная система испытывает непереориентационный фазовый переход в $\Phi M_{\|}$фазу.

Для определения линий фазового перехода сравним плотности свободных энергий в $\Phi M_{\|}$фазе и $N_{2}$ фазе. Из равенства свободных энергий следует, что линией фазового перехода является линия $J_{0}=K_{0}$.

В результате проведенного исследования нами построена фазовая диаграмма негейзенберговского магнетика с отрицательной межионной анизотропией, которая схематично приведена на рис. 5.

Фазовый переход $N_{2}-\Phi M_{\|}$, полученный в данной модели, не связан с изменением параметров межионной анизотропии, а определяется лишь соотношением 


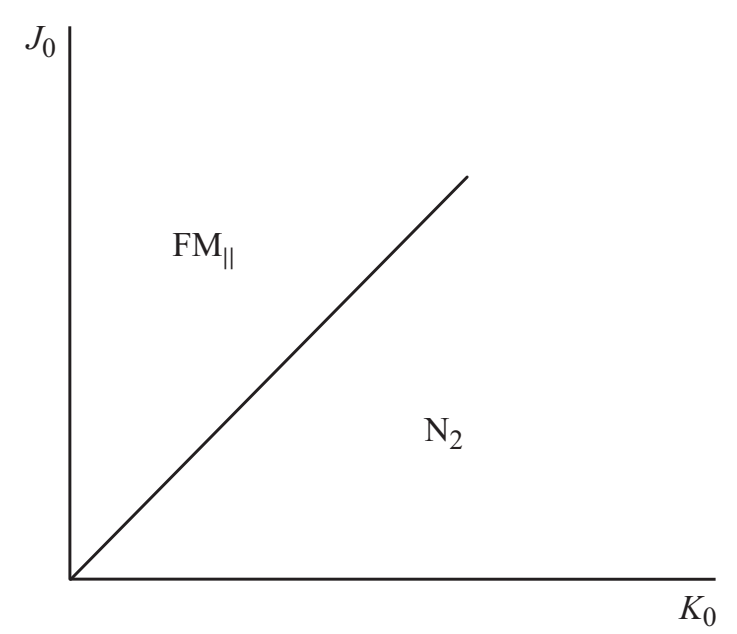

Рис. 5. Фазовая диаграмма обменно анизотропного изингоподобного негейзенберговского магнетика.

обменных интегралов. Это связано с тем, что данная модель представляет собой изингоподобный магнетик, в котором присутствует очень большая одноосная анизотропия (ось симметрии которой совпадает с осью квантования), которая ни с какими другими взаимодействиями не „конкурирует“. Переход же $N_{2}-\Phi M_{\|}$ обусловлен конкуренцией билинейного (изинговского) обменного взаимодействия с биквадратичным (также изинговским) обменным взаимодействием.

\section{5. Заключение}

Проведенные исследования позволяют выяснить влияние анизотропии обменного взаимодействия на формирование фазовых состояний и динамических свойств негейзенберговского магнетика со спином магнитного иона $S=1$. Так, при положительных значениях параметров межионной анизотропии система ведет себя как магнетик с двухосной анизотропией. Причем, поведение такого негейзенберговского магнетика имеет ряд особенностей. Прежде всего, это проявляется в том, что учет анизотропного обменного взаимодействия приводит к снятию вырождения по направлению вектора магнитного момента в ферромагнитной фазе и векторадиректора в нематическом состоянии. Сравнение с работами $[12,13]$, в которых также рассматривалось анизотропное обменное взаимодействие, показывает, что именно учет анизотропного биквадратичного обменного взаимодействия более сложного вида приводит к реализации угловых ферромагнитной и нематической фаз.

Совершенно иначе ведет себя рассматриваемая система в случае отрицательных параметров межионной анизотропии. Такая межионная анизотропия приводит к возникновению очень сильной эффективной одноосной анизотропии, параллельной оси квантования. Эту ситуацию можно рассматривать как изинговскую систему, аналогичную модели Блюма-Эмери-Грифитса [11].
В этом случае в системе реализуются только два фазовых состояния - ферромагнитная фаза $\Phi M_{\|}$, с магнитным моментом, параллельном оси квантования, и нематическая $N_{2}$ фаза, геометрическим образом которой является бесконечно тонкий диск, ориентированный в плоскости $X O Y$. При этом параметры межионной анизотропии хотя и определяют симметрию системы, но не определяют линию фазового перехода, которая совпадает с линией фазового перехода ФМ-N изотропного спинового нематика [5].

\section{Список литературы}

[1] N. Papanikolaou. Nucl. Phys. B 305, 367 (1988).

[2] A.V. Chubukov. J. Phys. Condens. Matter 2, 1593 (1990).

[3] M. Arikawa, H. Tsunetsugu. JMMM 310, 1308 (2007).

[4] Tarun Grover, T. Senthil. Phys. Rev. Lett. 98, 247202 (2007).

[5] Yu.A. Fridman, O.A. Kosmachev, Ph.N. Klevets. JMMM 325, 125 (2013).

[6] J. Oitmaa, C.J. Hamer. Phys. Rev. B 87, 224431 (2013).

[7] В.М. Матвеев. ЖЭТФ 65, 1626 (1973).

[8] Э.Л. Нагаев. Магнетики со сложными обменными взаимодействиями. Наука, М. (1988).

[9] О.А. Космачев, Ю.А. Фридман, Е.Г. Галкина, Б.А. Иванов. ЖЭТФ 147, 320 (2015).

[10] Yu.A. Fridman, O.A. Kosmachev, A.K. Kolezhuk, B.A. Ivanov. Phys. Rev. Lett. 106, 097202 (2011).

[11] M. Blume, V.J. Emery, R.B. Griffiths. Phys. Rev. A 10, 1071 (1971).

[12] Yu.A. Fridman, O.A. Kosmachev, P.N. Klevets. JMMM 320, 435 (2008).

[13] Ю.А. Фридман, О.А. Космачев, Ф.Н. Клевец. ФНТ 32, 289 (2006).

[14] P.A. Sayko1, I.P. Shapovalov. JMMM 392, 134 (2015).

[15] I. Шаповалов. УФЖ 55, 307 (2010).

[16] K. Stevens. Proc. Phys. Soc. A 65, 209 (1952).

[17] В.М. Калита. ФТТ 33, 1940 (1991).

[18] Р.О. Зайцев. ЖЭТФ 68, 207 (1975).

[19] В.М. Калита, И.М. Иванова, В.М. Локтев. ТМФ 173, 333 (2012).

[20] Б.А. Иванов, Р.С. Химин. ЖЭТФ 131, 343 (2007).

[21] В.Г. Барьяхтар, В.Н. Криворучко, Д.А. Яблонский. Функции Грина в теории магнетизма. Наук. думка, Киев (1984).

[22] В.В. Вальков, Т.А. Валькова, С.Г. Овчинников. ЖЭТФ 88, 550 (1985).

[23] Ф.П. Онуфриева. ЖЭТФ 80, 2372 (1981).

[24] A.F. Andreev, I.A. Grishchuk. Sov. Phys. JETP 60, 267 (1984).

[25] П. Де Жен. Физика жидких кристаллов. Мир, М. (1977).

[26] В.М. Локтев, В.С. Островский. ФНТ 20, 983 (1994).

[27] Yu.A. Fridman, O.A. Kosmachev, Ph.N. Klevets. Eur. J. B 81, 185 (2011).

[28] I.P. Shapovalov. Ukr. J. Phys. 53, 651 (2008).

[29] М.Ю. Ковалевский, А.В. Глущенко. ФНТ 40, 560 (2014).

[30] В.В. Вальков, Г.Н. Мацулева, С.Г. Овчинников. ФТТ 31, 60 (1989).

[31] Ю.А. Фридман, О.А. Космачёв. ФНТ 27, 642 (2001).

[32] Ф.П. Онуфриева. ЖЭТФ 89, 2270 (1988).

[33] Ph.N. Klevets, O.A. Kosmachev, Yu.A. Fridman. JMMM 330, 91 (2013).

[34] A. Smerald, N. Shannon. Phys. Rev. B 88, 184430 (2013). 